GENERAL

\title{
GENERAL
}

Pasquale Giugliano, ${ }^{1}$ M.D.; Francesco Massoni, ${ }^{2}$ M.D., Ph.D.; Antonello Crisci, ${ }^{3}$ M.D.; and

Serafino Ricci, ${ }^{2}$ M.D.

\section{Death from Barotrauma Due to Compressed Air: A Medico-legal Analysis}

\begin{abstract}
Lesions of the digestive tract due to barotrauma resulting from compressed air application are not common, are rarely lethal, and largely affect the sigmoid and descending colon. Moreover, their pathogenic mechanism is a topic of discussion because these lesions have multiple characteristics. Here, the authors describe an autoptic case of death from lesions of the ascending and transverse segments, with perforations and bleeding suffusions as well as ischemic areas covered the colonic wall that was extremely thinned, congested, and hemorrhagic, with considerable flattening leading to disappearance of the mucosal folds and with numerous petechial hemorrhages. The pathological framework of lung congestion made it possible to identify the mechanism responsible for this death as depletion of the heart's pumping function, which contributed significantly to the acute respiratory failure due to respiratory distress as well as to reduced mobility of the diaphragm due to intestinal distension. Acute heart failure played an important role in this death.
\end{abstract}

KEYWORDS: forensic science, death, rare, barotrauma, compressed air, ascending colon, transverse colon

Injuries from barotrauma resulting from the placement of high-pressure air in the digestive tract are not particularly common. Indeed, these injuries have been described as "rare, unique, and traumatic intra-abdominal injury" (1).

Stone reported the first case of pneumatic rupture of the colon in 1904. Such injuries typically affect the sigmoid and descending colon (2).

Deaths are rarely reported (3), and those stemming from involvement of the ascending or transverse colon are least frequent (4). The first case of involvement of the ascending colon was reported in 1912 (5), and the first case involving the transverse colon was reported in 1914 (6).

Here, the authors present an autopsy report describing death from barotrauma as a result of compressed air application, causing lesions of the ascending and transverse colon.

\section{Case Report}

A 17-year-old woman (BMI 19.5) without any history of disease died, according to her partner, as a result of the voluntary application of a pressure regulator, which regulates compressed air, to the anus for a short moment two hours before her death. The pressure regulator was subsequently analyzed, and it was found to have an operating pressure ranging from a minimum of 8.5 bar to a maximum of 10.5 bar. As a consequence of

${ }^{1}$ A.O.R.N. Sant'Anna e San Sebastiano, Caserta, Italy.

${ }^{2}$ Department of Anatomy, Histology, Legal Medicine and Orthopedics, Sapienza University, Rome, Italy. Italy.

${ }^{3}$ Department of Medicine and Surgery, University of Salerno, Salerno,

Received 17 Nov. 2015; and in revised form 1 Dec. 2015; accepted 2 Jan. 2016. applying the regulator, the victim complained of violent abdominal pain. She refused to be brought to the hospital for fear of having to reveal what happened. When she finally agreed to go to the hospital, she was very cold and was suffering from tachycardia. Unfortunately, by the time she arrived at the hospital, it was too late: she was already dead.

During external examination, the only noteworthy finding was that the patient's abdomen was slightly bulbous. There were no signs of lesions in the anal area.

Examination of the internal organs revealed that the lung size and consistence had increased (right lung weight of $875 \mathrm{~g}$; left lung weight of $825 \mathrm{~g}$ ) and that the lungs were with crackling sounds. The large, medium, and small bronchi had a hyperemic mucosa. The parenchyma appeared congested in a full-thickness section, with frothy bleeding. Edema and congestion were found on histological examination (Fig. 1). The heart had a globular shape (weight of $255 \mathrm{~g}$, longitudinal diameter of $10 \mathrm{~cm}$, transverse diameter of $11 \mathrm{~cm}$, and anteroposterior diameter of $3.5 \mathrm{~cm}$ ). On opening of the coronaries, the lumen was patent, and the size was unchanged. On sectioning, the thickness of the left ventricular free wall was approximately $1.1 \mathrm{~cm}$, and the thickness of the septum was $1.2 \mathrm{~cm}$. Examination of the rest of the heart showed no abnormalities in the endocardium, valves, or chordae.

On opening the abdomen and peritoneum, free blood was found in the cavity, and perforations and bleeding suffusions as well as ischemic areas covered the colonic wall. These features appeared to extend in all directions but were especially present in the cecum and ascending colon (Fig. 2).

On inspection, it was found that the wall was extremely 3 thinned, congested, and hemorrhagic, with considerable flattening leading to disappearance of the mucosal folds and with numerous petechial hemorrhages. 


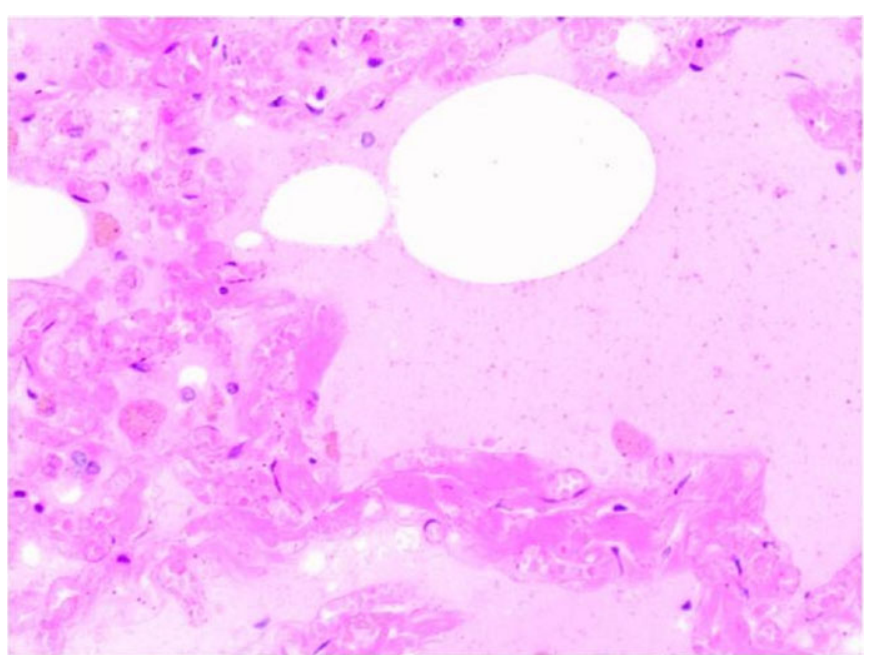

FIG. 1-Histological evidence of pulmonary edema and congestion.

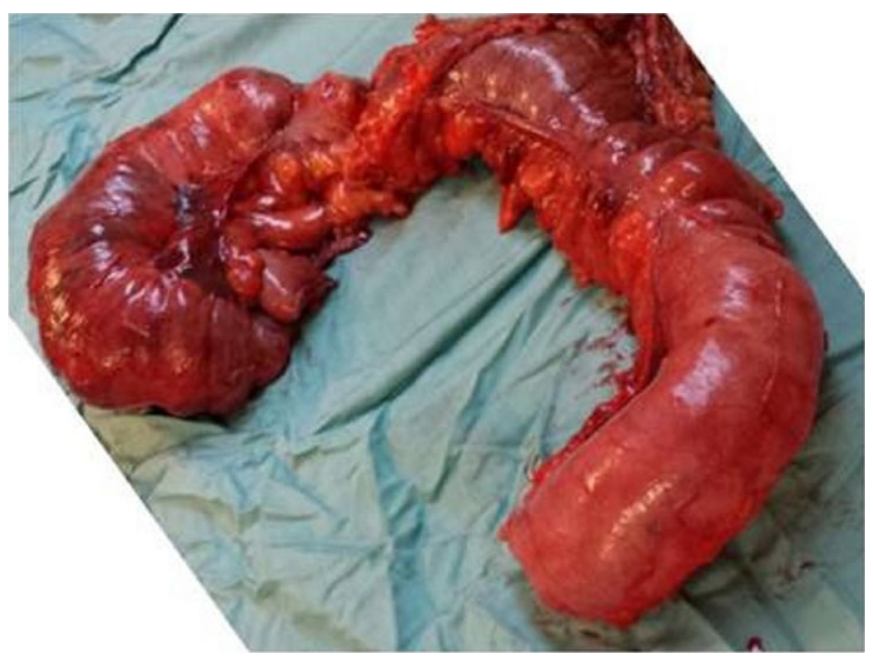

FIG. 2-Detail of the ascending and transverse colon after evisceration.

The thinning coupled with atrophy of the mucosal structure was histologically confirmed, as were edema, congestion, vascular ectasia, and hemorrhagic suffusions, with greater representation at the level of the ascending and transverse colon. At the subserosal site, necrosis, edema, microfocal areas of abscess, and optically empty focal areas containing pseudocysts were found.

Biochemical blood analysis and microbiological blood culture were negative.

\section{Discussion}

The release of high-pressure air into the digestive tract through the anal sphincter is described in the literature as having different detrimental and lethal effects. Its harmfulness is generally linked to a single incident that only rarely occurs due to improper use, an incident that had disastrous effects on the affected person (3).

The absence of injury at the anal level in the case described here suggests that the tube used for dispensing the air was introduced over the anal margin. However, certain cases are found in the literature in which there was no direct contact between the source of compressed air and the body of the victim. In fact, the clinical picture may occur even without insertion of the air tube into the anus (7).

Although the pressure of the employed port was known for the current case (8.5-10.5 bar), based on the clinical picture, it was not possible to determine the duration of action of the jet. In the literature, a pressure between 0.48 and 0.86 bar (8) is needed to cause acute injury in animals, although an average pressure of only 0.28 bar is required in humans (9). In contrast, the pressure of compressed air is $3.45-10.34$ bar, which is 10 30 times greater than the pressure required to affect the intestinal wall (of the four layers of the intestinal wall, the mucosa is the most resistant, yielding only after the muscularis and the serosa at a pressure of 0.28 bar (1). The severity of the resulting intestinal lesions is also related to the duration of dispensation. According to certain reports, it takes only a few seconds (even one or two seconds) to cause serious injury to the intestinal compartment (8), but there are also cases of delayed perforation, with appreciable results after five days (10).

Rupture usually occurs in the longitudinal direction, along the muscle fibers (11), but in contrast to previous reports in the literature, the cecal segment was the most affected tract in the case presented here. There are two possible explanations for this difference. First, the presence of the ileocecal valve may increase the intraluminal pressure gradient. Second, according to the law of Laplace, the intraluminal pressure required to distend the wall of a hollow tube is inversely proportional to its diameter; therefore, the cecal segment, with its large diameter, requires little pressure to undergo shock, with consequently increased mechanical stress and ischemia; these effects in turn cause injury.

The literature describes several mechanisms that lead to death in this context. Zechel identified the following two: shock from primary intestinal rupture and shock secondary to pneumoperitoneal shock (12). A greater number of mechanisms were hypothesized by Althoff, including acute gas embolism, acute fat embolism, acute respiratory failure, acute heart failure, and peritoneal shock (13).

In our case, the framework of pulmonary edema and congestion and the negative biochemical blood analysis and microbiological blood culture, which did not indicate a fat or gas embolism, permitted the recognition of an acute heart with overloading. A sharp increase in blood pressure can be attributed to the direct action of compression of the colon on the abdominal aorta, resulting in an increase in the systolic afterload $(14,15)$. Systolic overload of the heart contributes, through pulmonary hypertension, to accelerating functional impairment of breathing (respiratory distress) due to pneumoperitoneum and compression of the expansive loops on the diaphragm (1).

\section{Conclusion}

Barotrauma due to injection of compressed air into the digestive tract results in unpredictable and life-threatening disease that requires a timely diagnosis to enable the enactment of appropriate therapeutic measures. The circumstances related to the manner in which such an event occurs can adversely delay access to medical care, therefore representing a risk factor that can affect handling of the case.

\section{References}

1. Kim SJ, Ahn SI, Hong KC, Kim JS, Shin SH, Woo ZH. Pneumatic colonic rupture accompanied by tension pneumoperitoneum. Yonsei Med J 2000;41(4):533-5. 
2. Stone G. Rupture of bowel caused by compressed air. Lancet 1904;164 (4221):216-17.

3. Rayamane AP, Pradeepkumar MV. Accidental fatal lung injury by compressed air: a case report. Am J Forensic Med Pathol 2015;36(1):1-2.

4. Brown RK, Dwinelle JH. Rupture of the colon by compressed air: report of three cases. Ann Surg 1942;115(1):13-20.

5. Cotton FJ. Rupture of the bowel from compressed air. Boston Med Surg J 1912;166:562-3.

6. Bendixen PA, Blything JD. Pneumatic rupture of the bowel. Surg Gynecol Obstet 1914;18:73-7.

7. Losanoff JE, Richman BW, Jones JW. Colorectal blowout from compressed air. J Trauma 2002;53(5):1030. author reply 1030-1.

8. Andrews EW. Pneumatic rupture of the intestine, or new type of industrial accident. Surg Gynecol Obstet 1911;12:63-4.

9. Burt CAV. Pneumatic rupture of the intestinal canal. Arch Surg 1931;22:875-902.

10. Raina S, Machiedo GW. Multiple perforations of colon after compressed air injury. Arch Surg 1980;115(5):660-1.

11. Zunzunegui RG, Werner AM, Gamblin TC, Stephens JL, Ashley DW. Colorectal blowout from compressed air: case report. J Trauma 2002;52 (4):793-5.
12. Zechel GL. Pneumatic rupture of colon as an industrial injury. Ind Med Surg 1967;36:663-7.

13. Althoff H. Plötzlicher tod nach einer exposition mit druckluft. Monatsschr Unfallheilkd Versicher Versorg Verkehrsmed 1969;72:501-6.

14. Massoni F, Ricci S. Aortic valve sclerosis and atherosclerosis: autoptic report and review. Gazzetta Medica Italiana Archivio per le Scienze Mediche 2014;173(4):213-17.

15. Massoni F, Ricci P, Simeone C, Ricci S. Cardiac death in aortic valve sclerosis and coronary artery disease. An autopsy report. Acta Medica Mediterranea 2014;30(1):77-80.

Additional information and reprint requests:

Francesco Massoni, M.D., Ph.D.

Department of Anatomy, Histology, Legal Medicine and Orthopedics Sapienza University

Viale Regina Elena 336

Postal Code 00161

Rome

Italy

E-mail: francesco.massoni@uniroma1.it 